\title{
P01-7-5 Poster session
}

\section{Developing Capabilities in Practice for Clinical Pharmacology Training in the UK}

Jamie J Coleman ${ }^{1,2,3,5}$, Simon Hill ${ }^{4,5}$

\author{
${ }^{1}$ Institute of Clinical Sciences, University of Birmingham, UK, ${ }^{2}$ University Hospitals Birmingham NHS Foundation \\ Trust, UK, ${ }^{3}$ West Midlands Centre for Adverse Drug Reactions, Sandwell and West Birmingham Hospitals NHS Trust, \\ UK, ${ }^{4}$ The Newcastle upon Tyne Hospitals NHS Foundation Trust, UK, ${ }^{5}$ Specialty Advisory Committee for Clinical \\ Pharmacology and Therapeutics, Joint Royal College of Physicians Training Board, UK
}

A curriculum consists of the intended aims and objectives, content, experiences, outcomes and processes of a programme, including a description of the structure and expected methods of learning, teaching, feedback and supervision. In the UK, clinical pharmacology and therapeutics (CPT) trainees currently complete a five year training programme in combination with general internal medicine with three years of the curriculum dedicated to CPT competencies. The clinical pharmacology core module consists of 108 outcomes to be achieved during two years in addition to further 26-95 outcomes to be achieved in an advanced specialist CPT area (depending on choice - toxicology, research, hypertension and clinical trials). The final award of the Completion of Specialist Training is dependent on achieving these outcomes as evidenced by successful completion of supervised learning events set out in the curriculum against each outcome.

In current training there is a move away from a 'tick box' attitude of multiple outcomes towards a more efficient competency-based curricula, which is more flexible, less time-orientated and allows a trainee to show logical progression towards specialist accreditation.

The UK physicianly specialties are in the process of shifting from outcomes to develop a set of capabilities in practice (CiPs) for each specialty. These will work alongside a set of general professional capabilities common to all postgraduate doctors in training. CiPs are defined as a 'critical path to professional work that can be identified as a unit to be entrusted to a trainee once efficient competency has been reached'; this links with the concept of Entrustable Professional Activities (EPAs) which allow trainers to make competency-based decisions on the level of supervision required by trainees.

A working group of the Specialist Advisory Committee have drafted a short list of CiPs leading to a 'trusted decision' of competence for CPT trainees in the UK. Ten high level capabilities have been currently drafted for CPT specialist training and subject to ongoing consultation with specialists and trainees. At the congress we will present the latest draft of the CiPs as well as describe the evolving concept of curriculum development. 